\title{
Fluocinolone Acetonide
}

National Cancer Institute

\section{Source}

National Cancer Institute. Fluocinolone Acetonide. NCI Thesaurus. Code C29055.

The acetonide salt form of fluocinolone, a synthetic fluorinated corticosteroid with antiinflammatory, antipruritic and vasoconstrictive properties. Fluocinolone is a glucocorticoid receptor agonist that binds to cytoplasmic glucocorticoid receptors and subsequently translocates to the nucleus where it initiates the transcription of glucocorticoid-responsive genes such as lipocortins. Lipocortins inhibit phospholipase A2, thereby blocking the release of arachidonic acid from membrane phospholipids and preventing the synthesis of prostaglandins and leukotrienes, both are potent mediators of inflammation. Fluocinolone exerts its vasoconstrictive effect through inhibition of nitric oxide synthase, thereby blocking nitric oxide production and effectively diminishing the effect of nitric oxide on vascular smooth muscles leading to reduced blood flow. 